\title{
Bose-Einstein Condensation of Dark Matter Axions
}

\author{
P. Sikivie and Q. Yang \\ Department of Physics, University of Florida, Gainesville, FL 32611, USA
}

\begin{abstract}
We show that cold dark matter axions thermalize and form a Bose-Einstein condensate. We obtain the axion state in a homogeneous and isotropic universe, and derive the equations governing small axion perturbations. Because they form a BEC, axions differ from ordinary cold dark matter in the non-linear regime of structure formation and upon entering the horizon. Axion BEC provides a mechanism for the production of net overall rotation in dark matter halos, and for the alignment of cosmic microwave anisotropy multipoles.

PACS numbers: 95.35.+d
\end{abstract}

Several authors have proposed that the dark matter of the universe is a Bose-Einstein condensate (BEC) [1, 2]. The axion is sometimes mentioned in this context. Indeed the axion is a boson and a cold dark matter candidate, and cold dark matter axions are known to have huge phase space density. But, as far as we are aware, it has never been shown that dark matter axions form a BEC. Their phase space density is certainly large enough but they will only form a BEC if they reach thermal equilibrium. This may see unlikely because the axion is very weakly coupled. Below we find that dark matter axions do form a BEC, marginally because of their self interactions but certainly as a result of their gravitational interactions. No special assumptions are required.

Shortly after the Standard Model of elementary particles was established, the axion was postulated [3] to explain why the strong interactions conserve the discrete symmetries $\mathrm{P}$ and $\mathrm{CP}$. For the purposes of this paper the action density for the axion field $\varphi(x)$ may be taken to be

$$
\mathcal{L}_{a}=-\frac{1}{2} \partial_{\mu} \varphi \partial^{\mu} \varphi-\frac{1}{2} m^{2} \varphi^{2}+\frac{\lambda}{4 !} \varphi^{4}-\ldots
$$

where $m$ is the axion mass. The self-coupling strength is

$$
\lambda=\frac{m^{2}}{f^{2}} \frac{m_{d}^{3}+m_{u}^{3}}{\left(m_{d}+m_{u}\right)^{3}} \simeq 0.35 \frac{m^{2}}{f^{2}}
$$

in terms of the axion decay constant $f$ and the masses $m_{u}$ and $m_{d}$ of the up and down quarks. In Eq. (11), the dots represent higher order axion self-interactions and interactions of the axion with other particles. All axion couplings and the axion mass

$$
m \simeq 6 \cdot 10^{-6} \mathrm{eV} \frac{10^{12} \mathrm{GeV}}{f}
$$

are inversely proportional to $f$. $f$ was first thought to be of order the electroweak scale, but its value is in fact arbitrary [4]. However, the combined limits from unsuccessful searches in particle and nuclear physics experiments and from stellar evolution require $f \gtrsim 3 \cdot 10^{9} \mathrm{GeV}[5]$.

Furthermore, an upper limit $f \lesssim 10^{12} \mathrm{GeV}$ is provided by cosmology because light axions are abundantly produced during the QCD phase transition [6]. In spite of their very small mass, these axions are a form of cold dark matter. Indeed, their average momentum at the QCD epoch is not of order the temperature $(\mathrm{GeV})$ but of order the Hubble expansion rate $\left(3 \cdot 10^{-9} \mathrm{eV}\right)$ then. In case inflation occurs after the Peccei-Quinn phase transition their average momentum is even smaller because the axion field gets homogenized during inflation. For a detailed discussion see ref. [7]. In addition to this cold axion population, there is a thermal axion population with average momentum of order the temperature.

The non-perturbative QCD effects that give the axion its mass turn on at a temperature of order $1 \mathrm{GeV}$. The critical time, defined by $m\left(t_{1}\right) t_{1}=1$, is $t_{1} \simeq$ $2 \cdot 10^{-7} \sec \left(f / 10^{12} \mathrm{GeV}\right)^{\frac{1}{3}}$. Cold axions are the quanta of oscillation of the axion field that result from the turn on of the axion mass. They have number density

$$
n(t) \sim \frac{4 \cdot 10^{47}}{\mathrm{~cm}^{3}}\left(\frac{f}{10^{12} \mathrm{GeV}}\right)^{\frac{5}{3}}\left(\frac{a\left(t_{1}\right)}{a(t)}\right)^{3}
$$

where $a(t)$ is the cosmological scale factor. Because the axion momenta are of order $\frac{1}{t_{1}}$ at time $t_{1}$ and vary with time as $a(t)^{-1}$, the velocity dispersion of cold axions is

$$
\delta v(t) \sim \frac{1}{m t_{1}} \frac{a\left(t_{1}\right)}{a(t)}
$$

if each axion remains in whatever state it is in, i.e. if axion interactions are negligible. Let us refer to this case as the limit of decoupled cold axions. If decoupled, the average state occupation number of cold axions is

$$
\mathcal{N} \sim n \frac{(2 \pi)^{3}}{\frac{4 \pi}{3}(m \delta v)^{3}} \sim 10^{61}\left(\frac{f}{10^{12} \mathrm{GeV}}\right)^{\frac{8}{3}} .
$$

Clearly, the effective temperature of cold axions is much smaller than the critical temperature

$$
T_{\mathrm{c}}=\left(\frac{\pi^{2} n}{\zeta(3)}\right)^{\frac{1}{3}} \simeq 300 \mathrm{GeV}\left(\frac{f}{10^{12} \mathrm{GeV}}\right)^{\frac{5}{9}} \frac{a\left(t_{1}\right)}{a(t)}
$$

for BEC. Axion number violating processes, such as their decay to two photons, occur only on time scales vastly longer than the age of the universe. The only condition 
for axion BEC that is not manifestly satisfied is thermal equilibrium.

Axions are in thermal equilibrium if their relaxation rate $\Gamma$ is large compared to the Hubble expansion rate $H(t)=\frac{1}{2 t}$. At low phase space densities, the relaxation rate is of order the particle interaction rate $\Gamma_{s}=n \sigma \delta v$ where $\sigma$ is the scattering cross-section. The cross-section for $\varphi+\varphi \rightarrow \varphi+\varphi$ scattering due to axion self interaction is in vacuum

$$
\sigma_{0}=\frac{1}{64 \pi} \frac{\lambda^{2}}{m^{2}} \simeq 1.5 \cdot 10^{-105} \mathrm{~cm}^{2}\left(\frac{m}{10^{-5} \mathrm{eV}}\right)^{6} .
$$

If one substitutes $\sigma_{0}$ for $\sigma, \Gamma_{s}$ is found much smaller than the Hubble rate, by many orders of magnitude. However, in the cold axion fluid background, the scattering rate is enhanced by the average quantum state occupation number of both final state axions, $\sigma \sim \sigma_{0} \mathcal{N}^{2}$, because energy conservation forces the final state axions to be in highly occupied states if the initial axions are in highly occupied states. In that case, the relaxation rate is multiplied by one factor of $\mathcal{N}[8]$

$$
\Gamma \sim n \sigma_{0} \delta v \mathcal{N} .
$$

Combining Eqs. (4]6[8), one finds $\Gamma\left(t_{1}\right) / H\left(t_{1}\right) \sim \mathcal{O}(1)$, suggesting that cold axions thermalize at time $t_{1}$ through their self interactions, but only barely so.

It may seem surprising that the huge and tiny factors on the RHS of Eq. (9) cancel each other. In fact the cancellation is not an accident. Consider a generic axion-like particle (ALP) whose mass $m$ and decay constant $f$ are unrelated to each other. Its self interaction coupling strength $\lambda \sim \frac{m^{2}}{f^{2}}$. Cold ALPs appear at a time $t_{1} \sim \frac{1}{m}$ with number density $n\left(t_{1}\right) \sim f^{2} m$, and velocity dispersion $\delta v\left(t_{1}\right) \sim 1$. Substituting these estimates in Eqs. (6), (8) and (9), one finds that the thermalization rate is of order the Hubble rate at $t_{1}$, for all $f$ and $m$.

A critical aspect of axion BEC phenomenology is whether the BEC continues to thermalize after it has formed. Axion BEC means that (almost) all axions go to one state. However, only if the BEC continually rethermalizes does the axion state track the lowest energy state.

The particle kinetic equations that yield Eq. (9) are valid only when the energy dispersion $\frac{1}{2} m(\delta v)^{2}$ is larger than the thermalization rate [8]. After $t_{1}$ this condition is no longer satisfied. One enters then a regime where the relaxation rate due to self interactions is of order

$$
\Gamma_{\lambda} \sim \lambda n m^{-2} .
$$

$\Gamma_{\lambda}(t) / H(t)$ is of order one at time $t_{1}$ but decreases as $t a(t)^{-3}$ afterwards. Hence, self interactions are insufficient to cause axion BEC to rethermalize after $t_{1}$ even if they cause axion BEC at $t_{1}$. However gravitational interactions, which are long range, come in to play. The relaxation rate due to gravitational interactions is of order

$$
\Gamma_{\mathrm{g}} \sim G n m^{2} \ell^{2}
$$

where $\ell \sim(m \delta v)^{-1}$ is the correlation length. $\Gamma_{\mathrm{g}}(t) / H(t)$ is of order $4 \cdot 10^{-8}\left(f / 10^{12} \mathrm{GeV}\right)^{\frac{2}{3}}$ at time $t_{1}$ but grows as $t a^{-1}(t) \propto a(t)$. Thus gravitational interactions cause the axions to thermalize and form a BEC when the photon temperature is of order $100 \mathrm{eV}\left(f / 10^{12} \mathrm{GeV}\right)^{\frac{1}{2}}$.

The process of axion Bose-Einstein condensation is constrained by causality. We expect overlapping condensate patches with typical size of order the horizon. As time goes on, say from $t$ to $2 t$, the axions in $t$-size condensate patches rethermalize into $2 t$-size patches. The correlation length is then of order the horizon at all times, implying $\delta v \sim \frac{1}{m t}$ instead of Eq. (5), and $\Gamma_{\mathrm{g}} / H \propto t^{3} a^{-3}(t)$ after the BEC has formed. Therefore gravitational interactions rethermalize the axion BEC on ever shorter time scales compared to the age of the universe.

We now consider what implications axion BEC has for observation. The axion field may be expanded in modes labeled $\vec{\alpha}$ :

$$
\varphi(x)=\sum_{\vec{\alpha}}\left[a_{\vec{\alpha}} \Phi_{\vec{\alpha}}(x)+a_{\vec{\alpha}}^{\dagger} \Phi_{\vec{\alpha}}^{\star}\right]
$$

where the $\Phi_{\vec{\alpha}}(x)$ are the positive frequency c-number solutions of the Heisenberg equation of motion for the axion field

$$
D^{\mu} D_{\mu} \varphi(x)=g^{\mu \nu}\left[\partial_{\mu} \partial_{\nu}-\Gamma_{\mu \nu}^{\lambda} \partial_{\lambda}\right] \varphi(x)=m^{2} \varphi(x),
$$

and the $a_{\vec{\alpha}}$ and $a_{\vec{\alpha}}^{\dagger}$ are creation and annihilation operators satisfying canonical commutation relations. We neglect the self-interaction term $-\frac{1}{6} \lambda \varphi^{3}$, which would otherwise appear on the RHS of Eq. (13), because it is of order $\frac{\rho}{f^{2}} \varphi$, where $\rho$ is the axion density, and hence smaller by the factor $\left(\frac{a\left(t_{1}\right)}{a(t)}\right)^{3} \frac{t}{t_{1}}$ than the relevant terms (of order $\left.\frac{m}{t} \varphi\right)$ in that equation. When the self-interactions are included, one finds an instability in the axion BEC towards the formation of droplets. The analog of the sound speed [9] is imaginary in this case because the self interaction is attractive. However, the rate of droplet formation is negligibly small compared to the Hubble rate. The gravitational forces always dominate over the self interactions except briefly after the cold axions first appear at time $t_{1}$

Except for a tiny fraction, all cold axions go to a single state which we label $\vec{\alpha}=0$. The corresponding $\Phi_{0}(x)$ is the axion wavefunction. In the spatially flat, homogeneous and isotropic Robertson-Walker space-time,

$$
\Phi_{0}=\frac{A}{a(t)^{\frac{3}{2}}} e^{-i m t}
$$

where $A$ is a constant. The state of the axion field is $\left|N>=(1 / \sqrt{N !})\left(a_{0}^{\dagger}\right)^{N}\right| 0>$ where $\mid 0>$ is the empty state, defined by $a_{\vec{\alpha}} \mid 0>=0$ for all $\vec{\alpha}$, and $N$ is the number of axions. The expectation value of the stressenergy-momentum tensor is

$$
\begin{aligned}
<N\left|T_{\mu \nu}\right| N> & =N\left[\partial_{\mu} \Phi_{0}^{*} \partial_{\nu} \Phi_{0}\right. \\
& \left.+\partial_{\nu} \Phi_{0}^{*} \partial_{\mu} \Phi_{0}+g_{\mu \nu}\left(-\partial_{\lambda} \Phi_{0}^{*} \partial^{\lambda} \Phi_{0}-m^{2} \Phi_{0}^{*} \Phi_{0}\right)\right]
\end{aligned}
$$


Again we neglect the self-interaction term.

Consider first the behavior of axion BEC in a flat Minkowski space-time. Since the axions are nonrelativistic, $\Phi_{0}(x)=e^{-i m t} \Psi(x)$ with $\Psi(x)$ slowly varying. Neglecting terms of order $\frac{1}{m} \partial_{t}$ compared to terms of order one, Eq. (13) becomes the Schrödinger equation:

$$
i \partial_{t} \Psi=-\frac{\nabla^{2}}{2 m} \Psi
$$

It is useful [10] to write the wavefunction as

$$
\Psi(\vec{x}, t)=\frac{1}{\sqrt{2 m N}} B(\vec{x}, t) e^{i \beta(\vec{x}, t)} .
$$

In terms of $B(\vec{x}, t)$ and $\beta(\vec{x}, t)$ the energy and momentum densities are $(j, k=1,2,3) T_{00} \equiv \rho=m(B(\vec{x}, t))^{2}$ and $T_{0 j} \equiv-\rho v_{j}=-(B(\vec{x}, t))^{2} \partial_{j} \beta$, in the non-relativistic limit. The velocity field is therefore $\vec{v}(\vec{x}, t)=\frac{1}{m} \vec{\nabla} \beta(\vec{x}, t)$ [10]. Eq. (16) implies the continuity equation and the equation of motion

$$
\partial_{t} v^{k}+v^{j} \partial_{j} v^{k}=-\vec{\nabla} q
$$

where

$$
q(\vec{x}, t)=-\frac{\nabla^{2} \sqrt{\rho}}{2 m^{2} \sqrt{\rho}}
$$

Following the motion, the stress tensor is

$$
T_{j k}=\rho v_{j} v_{k}+\frac{1}{4 m^{2}}\left(\frac{1}{\rho} \partial_{j} \rho \partial_{k} \rho-\delta_{j k} \nabla^{2} \rho\right) \quad .
$$

For ordinary cold dark matter $(\mathrm{CDM})$ the last terms on the RHS of Eqs. (18) and (20) are absent.

To compare axion BEC with CDM we divide the observations into three arenas: 1 ) the behaviour of density perturbations on the scale of the horizon, 2) their behaviour during the linear regime of evolution within the horizon, and 3) their behaviour during the non-linear regime. We first discuss arena 2 where CDM provides a very successful description. Neglecting second order terms, the perturbation in the stress tensor implied by Eq. (20) is

$$
\delta T_{j k}=-\delta_{j k} \frac{\rho_{0}(t)}{4 m^{2}} \nabla^{2} \delta(\vec{x}, t)
$$

where $\rho_{0}(t)$ is the unperturbed axion density and $\delta(\vec{x}, t) \equiv \frac{\delta \rho(\vec{x}, t)}{\rho_{0}(t)}$. Because the RHS of Eq. (21) is proportional to the Kronecker symbol and the RHS of Eq. (18) is a gradient, vector and tensor perturbations are not affected by the additional forces associated with the axion BEC. Only the scalar perturbations are affected. The scalar perturbations are conveniently described in conformal Newtonian gauge [11] where the metric is

$$
d s^{2}=-(1+2 \psi(\vec{x}, t)) d t^{2}+a(t)^{2}(1+2 \phi(\vec{x}, t)) d \vec{x} \cdot d \vec{x} .
$$

Conservation of energy and momentum in this background implies the first order equations

$$
\begin{aligned}
\partial_{t} \delta+\frac{1}{a} \vec{\nabla} \cdot \vec{v} & =-3 \partial_{t} \phi+\frac{3 H}{4 m^{2} a^{2}} \nabla^{2} \delta \\
\partial_{t} \vec{v}+H \vec{v} & =-\frac{1}{a} \vec{\nabla} \psi+\frac{1}{4 m^{2} a^{3}} \vec{\nabla} \nabla^{2} \delta
\end{aligned}
$$

where $H=\frac{1}{a} \frac{d a}{d t}$. The equations for CDM are recovered by letting $m \rightarrow \infty$. The RHS of Einstein's equations are modified by the addition of $\delta T_{j k}$ to the stress tensor, but this modification does not play a role in our discussion because it is suppressed, relative to the leading terms, by the factor $\left(\frac{k_{\mathrm{ph}}}{m}\right)^{2}$, where $k_{\mathrm{ph}}$ is the physical wavevector of the perturbation.

It is clear from Eqs. (23) that axion BEC differs from CDM on small scales only. For scales that are well within the horizon $\left(k_{\mathrm{ph}}>>H\right)$, Eqs. (23) plus Einstein's equations imply

$$
\partial_{t}^{2} \delta+2 H \partial_{t} \delta-\left(4 \pi G \rho_{0}-\frac{k^{4}}{4 m^{2} a^{4}}\right) \delta=0
$$

for the Fourier components $\delta(\vec{k}, t)$ of $\delta(\vec{x}, t) . \quad \vec{k}=a \vec{k}_{\mathrm{ph}}$ is co-moving wavevector. We assumed $\phi=-\psi$ which is almost always the case [11] and certainly valid during the matter dominated era. Eq. (24) shows that the axion BEC has Jeans length

$$
\begin{aligned}
k_{\mathrm{J}}^{-1} & =\left(16 \pi G \rho m^{2}\right)^{-\frac{1}{4}} \\
& =1.02 \cdot 10^{14} \mathrm{~cm}\left(\frac{10^{-5} \mathrm{eV}}{m}\right)^{\frac{1}{2}}\left(\frac{10^{-29} \mathrm{~g} / \mathrm{cm}^{3}}{\rho}\right)^{\frac{1}{4}}(25)
\end{aligned}
$$

The Jeans length is small compared to the smallest scales $(\sim 100 \mathrm{kpc})$ for which we have observations on the behavior of density perturbations in the linear regime. Thus axion BEC and CDM are indistinguishable in arena 2 on all scales of observational interest.

In the non-linear regime of structure formation (arena 3 ) and in the absence of rethermalization, the relevant equations are

$$
\begin{array}{r}
\partial_{t} \rho+\vec{\nabla} \cdot(\rho \vec{v})=0 \quad, \quad \vec{\nabla} \times \vec{v}=0 \\
\partial_{t} \vec{v}+(\vec{v} \cdot \vec{\nabla}) \vec{v}=-\vec{\nabla} \psi-\vec{\nabla} q .
\end{array}
$$

Eqs. (26) are equivalent to the Schrödinger equation for particles in a Newtonian gravitational field. Axion BEC and CDM differ in that the $-\vec{\nabla} q$ term is absent from the force law for CDM. However, as was shown by numerical simulation [12], and as is expected from the WKB approximation, the differences occur only on length scales smaller than the de Broglie wavelength. Since the axion de Broglie wavelength (of order 10 meters in galactic halos) is negligbly small compared to all length scales of observational interest, we again find that axion BEC and CDM are indistinguishable when there is no rethermalization of the BEC. 
However, we found that gravitational interactions do rethermalize the axion $\mathrm{BEC}$ continually so that the axion state tracks the lowest energy state. This is relevant to the angular momentum distribution of dark matter axions in galactic halos. The angular momentum of galaxies is caused by the gravitational torque of nearby galaxies early on when protogalaxies are still close to one another [13]. The angular momentum distribution acquired by the dark matter particles determines the structure of the inner caustics that they form in galactic halos [14, 15]. If that distribution is characterized by net overall rotation, implying $\vec{\nabla} \times \vec{v} \neq 0$, the inner caustics are a set of "tricusp rings" 14]. If the velocity field is irrotational $(\vec{\nabla} \times \vec{v}=0)$, the inner caustics have a tent-like structure [15] quite distinct from that of tricusp rings. Evidence has been found for tricusp rings [16], as opposed to the tent-like caustics of the $\vec{\nabla} \times \vec{v}=0$ case. This raises a puzzle for CDM. Indeed one can show [15] that the velocity field of ordinary cold dark matter, such as WIMPs, remains irrotational as it is the result of gravitational forces which are proportional to the gradient of the Newtonian potential. The puzzle is solved if the dark matter is an axion BEC which rethermalizes while tidal torque is applied to it. Indeed, the lowest energy state for given total angular momentum is one in which each particle carries an equal amount of angular momentum. In that case there is net overall rotation. $\vec{\nabla} \times \vec{v} \neq 0$ is accomodated in the BEC through the appearance of vortices. The phenomenon is observed in quantum liquids and well understood [10].

Finally we consider the behaviour of density perturbations as they enter the horizon (arena 1). Here too axion BEC may differ from CDM. The CDM perturbations evolve linearly at all times. The axion BEC perturbations do not evolve linearly when they enter the horizon because the condensates which prevailed in neighboring horizon volumes rearrange themselves, through their gravitational interactions, into a new condensate for the expanded horizon volume. This produces local correlations between modes of different wavevector since the perturbation of wavevector $\vec{k}$, upon entering the horizon, is determined by the perturbations of wavevector say $\frac{1}{2} \vec{k}$ in its neighborhood. We propose this as a mechanism for the alignment of CMBR anisotropy multipoles [17] through the integrated Sachs-Wolfe (ISW) effect. Unlike CDM, the ISW effect is large in axion BEC because the Newtonian potential $\psi$ changes entirely after entering the horizon in response to the rearrangement of the axion BEC.

We conclude that a case can be made that a large fraction of the dark matter is axions. Although the QCD axion is best motivated, a large class of axion-like particles has the properties described here.
We thank Georg Raffelt, Larry Widrow and Edward Witten for alerting us to errors in earlier versions of our paper, and for stimulating comments. This work was supported in part by the U.S. Department of Energy under contract DE-FG02-97ER41029.

[1] S.-J. Sin, Phys. Rev. D50 (1994) 3650; J. Goodman, New Astronomy Reviews 5 (2000) 103; W. Hu, R. Barkana and A. Gruzinov, Phys. Rev. Lett. 85 (2000) 1158; J.-W. Lee and S. Lim, arXiv:0812.1342 and references therein; E.W. Mielke and J.A. Vélez Pérez, Phys. Lett. B671 (2009) 174.

[2] F. Ferrer and J.A. Grifols, JCAP 12 (2004) 012; C.G. Bohmer and T. Harko, JCAP 06 (2007) 025.

[3] R. D. Peccei and H. Quinn, Phys. Rev. Lett. 38 (1977) 1440 and Phys. Rev. D16 (1977) 1791; S. Weinberg, Phys. Rev. Lett. 40 (1978) 223; F. Wilczek, Phys. Rev. Lett. 40 (1978) 279.

[4] J. Kim, Phys. Rev. Lett. 43 (1979) 103; M. A. Shifman, A. I. Vainshtein and V. I. Zakharov, Nucl. Phys. B166 (1980) 493; A. P. Zhitnitskii, Sov. J. Nucl. 31 (1980) 260; M. Dine, W. Fischler and M. Srednicki, Phys. Lett. B104 (1981) 199.

[5] J.E. Kim, Phys. Rep. 150 (1987) 1; M.S. Turner, Phys. Rep. 197 (1990) 67; G.G. Raffelt, Phys. Rep. 198 (1990) 1.

[6] J. Preskill, M. Wise and F. Wilczek, Phys. Lett. B120 (1983) 127; L. Abbott and P. Sikivie, Phys. Lett. B120 (1983) 133; M. Dine and W. Fischler, Phys. Lett. B120 (1983) 137.

[7] P. Sikivie, Lect. Notes Phys. 741 (2008) 19.

[8] D.V. Semikoz and I.I. Tkachev, Phys. Rev. Lett. 74 (1995) 3093 and Phys. Rev. D55 (1997) 489. See also: S. Khlebnikov, Phys. Rev. A66 (2002) 063606 and references therein.

[9] N.N. Bogoliubov, J. Phys. (Moscow) 11 (1947) 23; T.D. Lee, K. Huang and C.N. Yang, Phys. Rev. 106 (1957) 1135; J. Bernstein and S. Dodelson, Phys. Rev. Lett. 66 (1991) 683.

[10] C.J. Pethik and H. Smith, Bose-Einstein Condensation in Dilute Gases, Cambridge University Press 2002.

[11] S. Dodelson, Modern Cosmology, Academic Press 2003.

[12] L.M. Widrow and N. Kaiser, Ap. J. 416 (1993) L71.

[13] P.J.E. Peebles, Ap. J. 155 (1969) 393.

[14] P. Sikivie, Phys. Rev. D60 (1999) 063501.

[15] A. Natarajan and P. Sikivie, Phys. Rev. D73 (2006) 023510.

[16] L.D. Duffy and P. Sikivie, Phys. Rev. D78 (2008) 063508, and references therein.

[17] M. Tegmark, A. de Oliveira-Costa and A. Hamilton, Phys. Rev. D68 (2003) 123523; A. de Oliveira-Costa, M. Tegmark, M. Zaldarriaga and A. Hamilton, Phys. Rev. D69 (2004) 063516; C.J. Copi, D. Huterer, D.J. Schwarz and G.D. Starkman, MNRAS 367 (2006) 79 and references therein. 